\title{
ACUTE KIDNEY INJURY
}

\section{Proximal tubule cells modulate inflammation after renal injury}

Expression of kidney injury molecule 1 (KIM-1) correlates with damage to the proximal tubule in acute kidney injury and chronic kidney disease (CKD), but little is known about the role of this protein in kidney injury. Now, researchers report that after acute injury, surviving proximal tubule cells downregulate the innate immune response and protect the kidney from further damage through KIM-1-mediated phagocytosis of apoptotic cells.

"Our laboratory originally identified KIM-1 as the most upregulated protein in the injured proximal tubule," say researchers Li Yang and Craig Brooks. "We also discovered that expression of KIM-1 on proximal tubule cells induces a remarkable phenotypeit transforms these epithelial cells into phagocytes. KIM-1 acts as a phosphatidylserine phagocytosis and scavenger receptor, which binds to lipids on the surface of apoptotic and necrotic cells, as well as to oxidized LDL.”

In their new study, the researchers investigated whether KIM-1-mediated phagocytosis could reduce inflammation and kidney damage after injury. They found that the renal tubules of mice that expressed a functionally deficient form of KIM-1

(KIM-1 $\left.{ }^{\Delta \text { mucin }}\right)$ contained greater numbers of apoptotic bodies and cellular debris after ischaemia/ reperfusion (I/R) than did those of wild-type mice. Further experiments using lysosomal inhibitors confirmed a role of KIM-mediated phagocytosis in the removal of apoptotic cells and debris after kidney injury.

Interestingly, the KIM- $1^{\Delta \text { mucin }}$ mice showed more severe renal dysfunction and tubulointerstitial damage after I/R - and poorer survival after cisplatin insult-than did wild-type controls. Moreover, the injured kidneys of KIM-1 ${ }^{\Delta \text { mucin }}$ mice showed increased mRNA levels of proinflammatory cytokines and chemokines, numbers of infiltrating granulocytes and macrophages, and nuclear factor $\kappa \mathrm{B}(\mathrm{NF}-\kappa \mathrm{B})$ activity compared with controls. "Inhibition of the phosphatidylinositol 3-kinase pathway prevented KIM-1mediated suppression of NF- $\kappa \mathrm{B}$ activity in wild-type cells," report Yang and Brooks. "Together our findings reveal a natural defence system against acute tubular injury, whereby proximal tubular cells act as phagocytes to suppress inflammation and prevent further renal damage."

The researchers suggest that modulation of KIM-1 activity could be a therapeutic option for the treatment of both acute and chronic kidney injury. "We expect activation of KIM-1 in the acute phase of kidney injury to block proinflammatory signalling and ameliorate disease, whereas inhibition of KIM-1 in the chronic phase would prevent progression of CKD to end-stage renal disease. Going forward, we plan to further elucidate the mechanism by which KIM-1 regulates inflammation, and the role that proximal tubule cells play in immune signalling, recruitment of immune cells and profibrotic signalling."

\section{Ellen F. Carney}

Original article Yang, L. et al. KIM-1-mediated phagocytosis reduces acute injury to the kidney. J. Clin. Invest. doi:10.1172/JCI75417

Further reading Ichimura, T. et al. Kidney injury molecule-1 is a phosphatidylserine receptor that confers a phagocytic phenotype on epithelial cells. J. Clin. Invest. 118, 1657-1668 (2008)

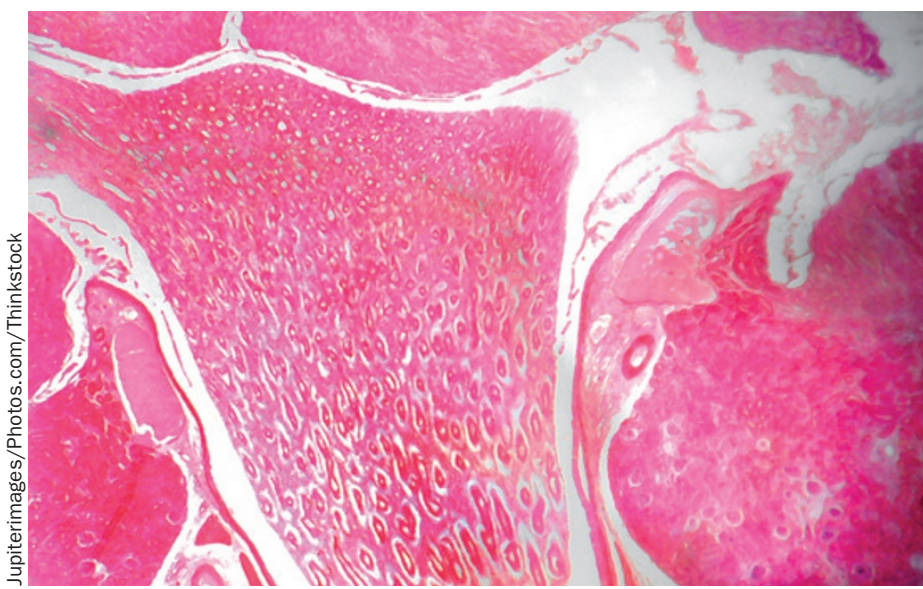

